\title{
Electron holography with variable magnification for semiconductor device characterization
}

\author{
Y.Y. Wang[a], A. Domenicucci[a], M. Kawasaki[b], J. Bruley[a], M. Gribelyuk[a], and J. Gaudiello[a] \\ a).IBM, Zip 40E, Hudson Valley Research Park, 2070 Route 52, Hopewell Jct., NY 12533, USA \\ b).JEOL USA, Inc., 11 Dearborn Road, Peabody, MA 01960, USA
}

In 1947, Dennis Gabor proposed a method of interference imaging for transmission electron microscopy (TEM), known as off-axis electron holography. Unlike conventional TEM imaging, where only the amplitude of the electron wave exiting the sample is recorded, off-axis electron holography produces both amplitude and phase information. The phase information is proportional to the mean inner potential of semiconductor material (or any other material) which can be related to the Fermi energy difference between p-doped (hole) and n-doped (electron) regions. Based on this principle, electron holography has been used to produce 2-D electrical junction images of semiconductor devices.[1]

There are three critical parameters in electron holography: the width, the spacing, and contrast of the interference fringe pattern, which determine the field of view (FOV), spatial resolution, and sensitivity of the resultant potential maps, respectively. In order to generate useful potential maps for the current generation of semiconductor devices, it is necessary (1) the fringe width be in the range of 100 to $1000 \mathrm{~nm}$ (2) the fringe spacing be between 0.5 and $10 \mathrm{~nm},(3)$ the fringe contrast be $10 \%-30 \%$, and (4) the width and spacing of the fringes be adjustable and optimized relative to device dimensions. In 1996, Lichte derived a pair of electron holography equations which indicated that the fringe spacing and width are inversely related to the magnification of the objective lens: the larger the magnification, the smaller the fringe spacing and width relative to the sample.[2] Based on Lichte's notation, we derived a third equation for fringe contrast.[3] The equation shows that the fringe contrast is mainly determined by biprism voltage and it is less sensitive to the magnification of objective lenses.

In the conventional TEM configuration, where the magnification of the objective lens is usually fixed and the total magnification is controlled by the projection lenses, the FOV and spatial resolution for electron holography is limited. In order to achieve FOV's and spatial resolution to be adjustable, a method of using the two objective lenses on a JEOL 2010F simultaneously was developed to provide a continuously varying the magnification of objective lenses at a fixed intermediate imaging plane.[3] This dual lens operation allows electron holography to be performed from low to high magnifications and provides the FOV's and fringe spacing necessary for two-dimensional (2-D) junction mapping of semiconductor devices.

Figure 1 illustrates the optical diagram of dual lens operation mode. The voltage of the first objective lens (OL) is set so that the position of the object is behind the first focal point $f_{1 \mathrm{OL}}$, but before the lens thereby forming a virtual image of the object. The second objective lens (OM) is used to project OL virtual image onto the intermediate image plane beyond the biprism as a real image. The magnification of the sample at the image plane, $M_{\mathrm{obj}}$, can be adjusted by varying the focal length of the first objective lens (OL) in conjunction with the focal length of the second objective lens (OM).

Figure 2 summarizes experimental results for fringe spacing $\left(\sigma_{\mathrm{obj}}\right)$ and width $\left(w_{\mathrm{obj}}\right)$ relative with OL excitation at a constant biprism voltage $(20 \mathrm{~V})$. The fringe spacing and width decrease as OL increases, but at different rates, which can be explained by geometric optics and Lichte's equation.[2,3] The contrast varies slightly with OL excitation (or magnification) for a constant biprism voltage, which is in agreement with the fringe contrast equation published in reference 3 .

The graphical results show that the range of values for field of view and fringe spacing necessary for semiconductor device characterization are achieved by dual lens operation. Moreover, although the fringe contrast is strongly dependent on the biprism voltage, it is only weakly dependent on the OL excitation. As a result, the fringe contrast can be optimized by varying the biprism voltage, while field of view and spatial resolution can be adjusted by the excitation of the objective lenses. In all, the data show that the dual lens mode leads to a large operational space for electron holography without sacrificing fringe contrast, which determines the signal to noise ratio in the amplitude and phase (potential) maps derived from the electron holograms.

To demonstrate the flexibility of the dual lens method, electron holograms were taken of pFET devices with poly silicon gate lengths of $220 \mathrm{~nm}$ and $70 \mathrm{~nm}$ on bulk Si and on silicon on insulator (SOI) substrates were obtained, respectively. Samples were prepared by mechanically polishing to an initial thickness of $\sim 400 \mathrm{~nm}$ and were ion milled to reduce the thickness to a final value of $\sim 200-300 \mathrm{~nm}$. The samples were then coated on one side with $\sim 20 \mathrm{~nm}$ of carbon to reduce the effects of charging. Two holograms were obtained during the operation using a biprism voltage of $20 \mathrm{~V}$ : one is the hologram with the sample and the other is the hologram without sample, known as a reference hologram. Phase and amplitude maps were obtained from these two holograms through Fast Fourier and inverse Fast Fourier 
Transformations (FFT) using Holoworks plug in to the Gatan Digital Micrograph software. Potential and amplitude maps were extracted from the phase map with a method described in reference 4 . Contour potential maps for the two devices are shown in Fig.3. In figure 3a, with $220 \mathrm{~nm}$ gate length, the holograms were formed at lower magnification $\left(M_{\mathrm{obj}}=5.7\right.$, OL value of $2.0 \mathrm{~V}$ on the JEOL 2010F) with fringe width and spacing of $800 \mathrm{~nm}$ and $2.5 \mathrm{~nm}$, respectively. For the device with $70 \mathrm{~nm}$ gate length (Fig. $3 \mathrm{~b})$, the holograms were formed at a higher magnification $\left(M_{\mathrm{obj}}=14.8, \mathrm{OL}\right.$ value of $3.5 \mathrm{~V}$ ) with fringe width and spacing as $575 \mathrm{~nm}$ and $1.4 \mathrm{~nm}$.

We would like to acknowledge the assistance and discussions provided by Dr. Y. Zhu and Dr. M. Schofield at Brookhaven National Lab and Dr. L. Gignac at IBM Watson Research Lab in the preliminary investigations of the subject. Mr. S. Klepeis and Mr. L. Kimball of IBM are acknowledged for their skillful work in preparing the TEM samples used in this study.

\section{Reference:}

1).W.D. Rau, P. Schwander, R.H. Baumann, F.H. W. Hoppner, A. Ourmazd, Physical Review Letter, 82, (1999), 2614; 2).H. Lichte, Ultramicroscopy 64 (1996), 79; 3).Y.Y. Wang, M. Kawaski, J. Bruley, M. Gribelyuk, A. Domenicucci, J. Gaudiello: Ultramicroscopy 101 (2004), 63; US patent application; 4).M.R. Mccartney, Ultramicroscopy, 53, (1994), 283.

\section{Figure captions:}

Fig.1: Optical ray diagram of dual lens system: The first objective lens forms a magnified virtual image and the second objective lens projected the virtual image onto the imaging plane.

Fig.2: Fringe spacing and width relative to the sample vs. OL excitation. As OL voltage increases and the magnification of the imaging lenses increases, both fringe spacing and width decrease but with different decreasing rate.

Fig.3: Potential maps of $220 \mathrm{~nm}$ gate device (fig.3a) and $70 \mathrm{~nm}$ gate devices on silicon on insulator (fig.3b). In fig.3b, BOX is defined as buried oxide $\left(\mathrm{SiO}_{2}\right)$ under the $\mathrm{Si}$ diffusion region.

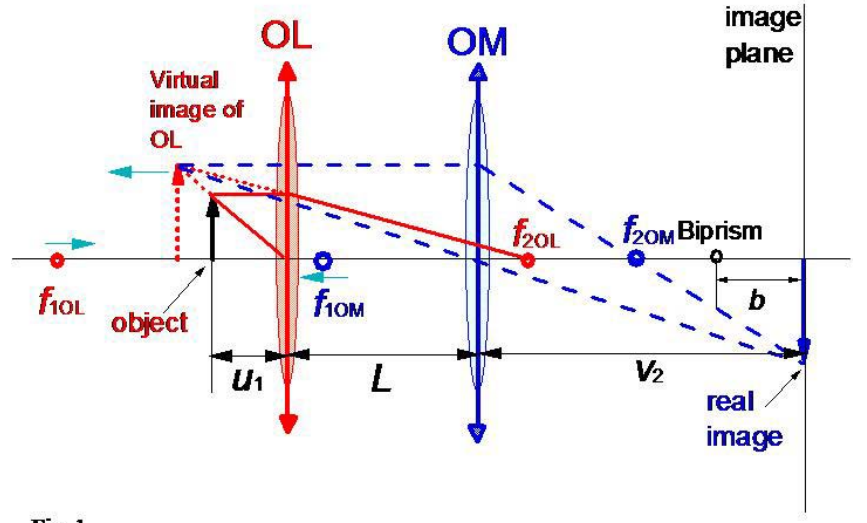

Fig. 1

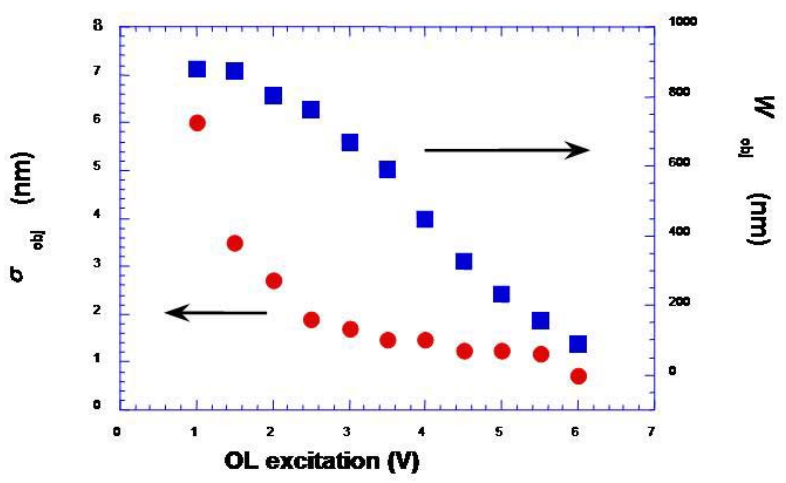

Fig. 2

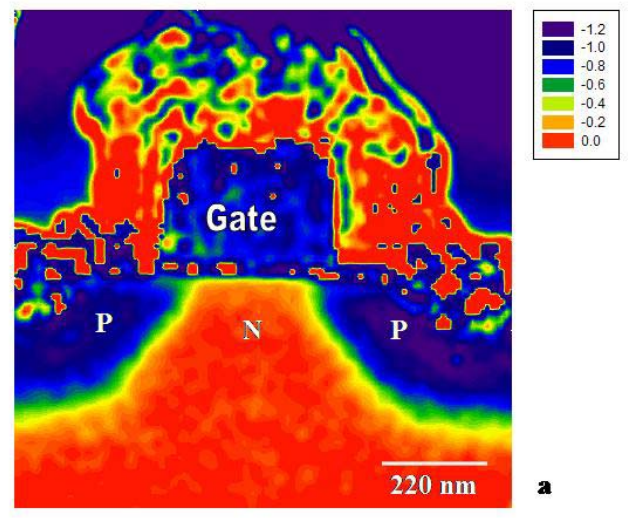

Figme 3a

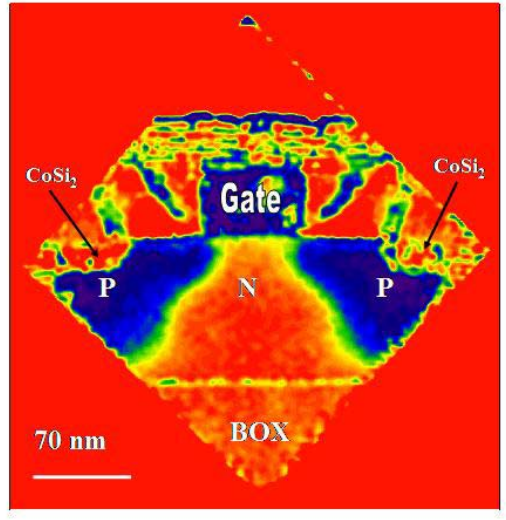

b

Figure 3b 